\title{
基于苯丙氨酸衍生物的小分子肽合成及抗肿瘤活性的研究
}

\author{
李洪宝 $^{a}$ 张 爽 ${ }^{b}$ 龚显峰 ${ }^{a}$ 侯广峰*,,$b$ \\ 于颖慧 ${ }^{a}$ 白有银 ${ }^{a}$ 高金胜*, $a, b$ \\ ( ${ }^{a}$ 黑龙江大学化学化工与材料学院 哈尔滨 150080) \\ ( ${ }^{b}$ 黑龙江大学农药工程中心 哈尔滨 150080)
}

\begin{abstract}
摘要 为了寻找更高效、更经济的抗肿瘤药物, 以 $L$-苯丙氨酸为起始原料, 经氨基保护、酰胺缩合、脱保护、再缩合、 再脱保护、再缩合等多步反应, 设计并合成了 8 种新型苯丙氨酸衍生物的小分子肽, 并采用 FTIR、 ${ }^{1} \mathrm{H} N M R 、{ }^{13} \mathrm{C} N M R$ 、 HRMS 和元素分析等测试方法对其结构进行确认. 采用溴化噻唑蓝四氮唑法(MTT)比色法对所有目标化合物进行了抗 肿瘤活性测试, 测试结果表明, 所有化合物对肝癌细胞 SMC7721 均有一定的抑制作用, 其中化合物 $\mathbf{5 e}$ 和 $8 \mathbf{b}$ 对肝癌细 胞的抑制作用最强.
\end{abstract}

关键词＼cjkstart苯丙氨酸; 小分子肽; 抗肿瘤活性测试; 合成

\section{Synthesis and Antitumor Activity of Small Peptides Based on Phenylalanine Derivatives}

\author{
Li, Hongbao $^{a}$ \\ Zhang, Shuang ${ }^{a}$ \\ Gong, Xianfeng ${ }^{a}$ \\ Hou, Guangfeng ${ }^{*, a, b}$ \\ Yu, Yinghui ${ }^{a}$ \\ Bai, Youyin $^{a}$ \\ Gao, Jinsheng*,a,b \\ $\left({ }^{a}\right.$ School of Chemistry and Materials Science, Heilongjiang University, Harbin 150080) \\ ( ${ }^{b}$ Engineering Research Center of Pesticide, Heilongjiang University, Heilongjiang University, Harbin 150080)
}

\begin{abstract}
With the aim of obtaining potential antitumor candidates with more efficiency and more economic value, eight new small peptides based on phenylalanine derivatives were designed and synthesized. The target products were obtained via amino protection, amide condensation, amino deprotection, condensation, deprotection and condensation reactions with $L$-phenylalanine used as the starting material. The structures of target products are confirmed by FTIR, ${ }^{1} \mathrm{H}$ NMR, ${ }^{13} \mathrm{C}$ NMR, HRMS and elemental analysis. The antitumor activities of all target compounds are evaluated by thiazolyl blue tetrazolium bromide (MTT) method. The results showed that all compounds exhibited moderate antitumor activities against hepatoma carcinoma cell SMC7721. Compounds $\mathbf{5 e}$ and $\mathbf{8 b}$ exhibited the strongest inhibitory activities against hepatoma carcinoma cell, which could serve as lead compounds and provided valuable information for the further development of new antitumor agents. Keywords phenylalanine; small peptide; antitumor activity tests; synthesis
\end{abstract}

目前，癌症是世界公认的对人类生命危害最严重、 最常见的顽症之一, 成为继心血管疾病之后的第二大杀 手. 随着人类生活习惯和生存环境的改变, 在一些不利 因素的作用下，恶性肿瘤的发病率正在逐年增加 ${ }^{[1,2]}$. 因 此, 抗肿瘤药物的研究受到了各国政府的高度重视, 其 中小分子肽类抗肿瘤药物因其具有分子量小、对细胞及 核酸的亲和性好、易穿透肿瘤细胞, 有利于药物在细胞
内更好的发挥作用等优点，而备受化学工作者的关 注 $^{[3,4]}$.

近年来, 人们通过从生物体中提取、肽库中篮选以 及化学合成等方法, 得到许多具有抗肿瘤活性的小分子 肽，例如胞壁酰二肽(MDP)及其衍生物，其可通过激活 $\mathrm{NK}$ 细胞、淋巴细胞和巨噬细胞并促使炎症细胞因子释 放而达到抗肿瘤作用 ${ }^{[5]}$. 又例如 RGD 三肽，通过抑制整

\footnotetext{
*E-mail: hougf@hlju.edu.cn; gaojins@hlju.edu.cn

Received May 4, 2015; revised June 26, 2015; published online July 2, 2015.

Project supported by the National Natural Science Foundation of China (Nos. 21371052), the Heilongjiang Postdoctoral Scientific Research Foundation (No. LBH-Q14138) and the Scientific Research Fund of Heilongjiang Provincial Education Department (No. 12531512).

国家自然科学基金(Nos. 21371052)、黑龙江省博士后科研启动基金(Nos. LBH-Q14138)及黑龙江省教育厅科学研究基金(No. 12531512)资助项目.
} 
合素介导的肿瘤细胞和细胞外基质的粘附, 来阻断肿瘤 细胞的浸润与侵袭, 从而达到抗肿瘤效果 ${ }^{[6]}$. $L$-苯丙氨 酸作为一种合成小分子肽的重要中间体, 是人体必需氨 基酸之一, 其本身对肿瘤细胞的生长起着十分重要的作 用, 如 Eagle 等 ${ }^{[7]}$ 用细胞培养的方法, 发现肿瘤细胞对 $L$-苯丙氨酸的需要量是正常细胞的 $3 \sim 5.5$ 倍. 又如 $\mathrm{McCoy}$ 等 ${ }^{[8]}$ 从肿瘤细胞的培养基中除去 $L$-苯丙氨酸时, 发现细胞的生长受到了明显的抑制, 从而说明 $L$-苯丙氨 酸可以作为一种有效的药物载体. 事实上, 很多 $L$-苯丙 氨酸衍生物对肿瘤细胞都有抑制作用, 如 $L$-苯丙氨酸吡 咯衍生物 ${ }^{[9]} 、 L$-苯丙氨酸芳基呋喃衍生物 ${ }^{[10]} 、 L$-苯丙氨 酸磷酸衍生物 ${ }^{[11]}$ 以及 $L$-苯丙氨酸氮芥 ${ }^{[12]}$ 等. 然而, $L$-苯 丙氨酸衍生物的小分子肽类化合物在抗肿瘤方面还很 少有人报道, 我们课题组选取 $L$-苯丙氨酸、 $L$-丙氨酸和 $L$-缴氨酸与具有抗肿瘤活性的苯胺 ${ }^{[13]}$ 和具有生物活性 的氯甲酸异丙酯 ${ }^{[14]}$ 相结合, 利用活性基团叠加原理, 制 备了系列具有抗肿瘤活性的 $L$-苯丙氨酸衍生物的小分 子肽, 进而在一定程度上丰富了 $L$-苯丙氨酸衍生物抗肿 瘤活性的研究内容.

本文以 $L$-苯丙氨酸为主体, 合成了 8 种未被文献报 道的新型苯丙氨酸衍生物的二肽和三肽化合物. 在肽键 合成中，我们用三氯氧磷代替昂贵的传统缩合剂 (DCC/HOBT、CDI、EDCI、HATU 等), 降低了成本、 缩短了反应时间、提高了产率、简化了后处理的操作步 骤. 同时采用 FTIR、 ${ }^{1} \mathrm{H}$ NMR、 ${ }^{\mathrm{I}} \mathrm{C}$ NMR、HRMS 和元 素分析等测试方法对其结构进行确认. 本文还对其中 8 个目标化合物进行了抗肿瘤活性测试, 测试结果表明, 所有化合物对肝癌细胞 SMC7721 均有一定的抑制作用, 其中化合物 $5 \mathbf{e}$ 和 $8 \mathrm{~b}$ 对肝癌细胞的抑制作用最强.

\section{1 结果与讨论}

\section{1 合成}

本文合成路线如 Scheme 1 所示. 化合物 $\mathbf{1 a} \sim \mathbf{1 c}$ 是 由 $L$-氨基酸与氯甲酸异丙酯反应制得 ${ }^{[14]}$. 由于反应时 剧烈放热, 应在低温下滴加氯甲酸异丙酯, 避免杂质生 成降低产率. 本体系中使用氢氧化钠作碱, 不仅中和了 反应中生成的氯化氢, 而且可以与羧基成钠盐, 避免羧 基与氯甲酸异丙酯成酸酤, 确保反应的高收率. 化合物 $2 \mathbf{a}$ 和 $2 \mathbf{b}$ 的制备也是以 $L$-氨基酸为起始原料, 只是改为 与二碳酸二叔丁酯(Boc)反应. 此反应中改用三乙胺作 碱，对氨基酸的氨基进行去质子化，活化氨基，并且三 乙胺在反应中间体过程中拔去氮正离子上的氢，再同 $\mathrm{BocO}$ 负离子反应生成 $\mathrm{BocOH}$, 而 $\mathrm{BocOH}$ 不稳定, 生成 了 $\mathrm{CO}_{2}$ 和叔丁醇, 促进反应的正向进行, 最终得到产物 $\mathbf{2 a}$ 和 $\mathbf{2 b}$. 上述虽然都是氨基的酯基保护反应, 但反应条
件有很大不同，在制备 $\mathbf{2 a}$ 和 $\mathbf{2 b}$ 反应中，改用三乙胺作 碱产率较文献方法 $(78 \% \sim 87 \%)$ 有很大提高，均可达到 $93 \%$ 以上，达到了优化目的 ${ }^{[15]}$.

化合物 3a $\sim 3 b 、 5 a \sim 5 f$ 和 $6 \mathbf{6} \sim 6 \mathbf{b}$ 均是由酰胺脱水 缩合得到. 以三乙胺作碱, 三氯氧磷作缩合剂和催化剂. 首先，氨基酸酯 $1 \mathrm{a} \sim 1 \mathrm{c}$ 或 $2 \mathrm{a} \sim 2 \mathrm{~b}$ 的羧基与三氯氧磷反 应形成氨基酸-磷酸混酐中间体，另一分子 4-氟苯胺(4三氟甲氧基苯胺)或苯丙氨酸衍生物 $4 a \sim 4 b$ 的氨基会进 攻混酐羰基上的碳原子，形成了酰胺键，从而生成产品. 在上述反应过程中，严格控制滴加三氯氧磷时的温度， 保持在 $0{ }^{\circ} \mathrm{C}$ 以下, HPLC 跟踪监测, $2 \mathrm{~h}$ 反应完毕, 滴加 时温度过高和反应时间过长会有杂质生成，导致产品收 率降低. 在化合物 5a 5f 和 $6 \mathrm{a} \sim 6 \mathrm{~b}$ 的合成中，碱的用 量较 3a 3b 合成中要多, 碱比例过小时杂质增多, 产率 降低. 其中选用三氯氧磷作缩合剂和催化剂, 缩短了反 应时间、简便了后处理操作、并且提高了产率.

化合物 $4 a \sim 4 b$ 和 $7 a \sim 7 b$ 是由化合物 $3 a \sim 3 b$ 和 6a $\sim 6$ b 在酸性条件下脱去Boc 保护基制得. 我们选用二 氯甲烷(DCM)作溶剂，三氟乙酸(TFA)作脱保护剂，三 氟乙酸提供质子，进攻氨基酸中氮上的孤对电子，生成 碳酸酯中间体和异丁基正离子，碳酸酯中间体和异丁基 正离子均不稳定, 分解生成产物的三氟乙酸盐、二氧化 碳和异丁烯，调碱后可得到产品. 其中脱 Boc 时, 酸的 浓度必须严格控制，过低会延长反应时间并且 Boc 不能 完全脱掉, 过高会导致肽键断裂. 通过大量实验, 我们 确定化合物 $4 \mathbf{a} \sim \mathbf{4 b}$ 的最佳反应物料比为 $n_{\mathbf{4}}: n_{\mathrm{TFA}}$ : $n_{\mathrm{DCM}}=1: 6: 24.5$, 化合物 $7 \mathbf{a} \sim 7 \mathbf{b}$ 的最佳反应物料比为 $n_{4}: n_{\mathrm{TFA}}: n_{\mathrm{DCM}}=1: 5.5: 26.3$.

化合物 $8 \mathrm{a}$ 和 $8 \mathrm{~b}$ 的合成方法与 $\mathbf{5 a} \sim \mathbf{5 f}$ 的方法类似， 其中物料比有所增加，反应温度控制更低，反应时间有 所延长，全程通过 HPLC 监测确定最佳反应条件.

\section{2 图谱分析}

以目标化合物 $\mathbf{8 b}$ 为例. 红外谱图中, 在 3294, 2982, $1683,1651,1540,1267$ 和 $1224 \mathrm{~cm}^{-1}$ 附近有特征吸收峰; ${ }^{1} \mathrm{H}$ NMR 核磁共振谱图中, 异丙酯基和甲基上的氢原子 所处化学环境相似, 在 $\delta 1.15$ 处出现 $\mathrm{t}$ 峰(12H); 苯丙氨 酸中 $\mathrm{CH}_{2}$ 上的氢原子由于化学等价磁不等价, 在 $\delta$ 2.91 3.11 处出现多重峰 $(2 \mathrm{H})$, NHCOO 中氢原子由于异 丙氧甲酰基的供电子效应向高场移动，也在此处出峰; 肽链中的四个 $\mathrm{CH}$ 上氢原子分别在 $\delta 3.97 \sim 4.04,4.22 \sim$ $4.27,4.59 \sim 4.64,4.69 \sim 4.75$ 处出现多重峰, 表明了三个 氨基酸的连接情况; $\delta 7.18 \sim 7.32$ 处的多重峰 $(7 \mathrm{H})$ 和 $\delta$ 7.70 处的双峰 $(2 \mathrm{H})$ 为苯丙氨酸苯环和苯胺苯环的偶合 峰; $\delta 7.97$ 处双峰 $(1 \mathrm{H}), \delta 8.16$ 处双峰 $(1 \mathrm{H}), \delta 10.24$ 处单 峰 $(1 \mathrm{H})$ 为酰胺 $\mathrm{NH}$ 中氢原子, 也证明了产品的肽链结构; 


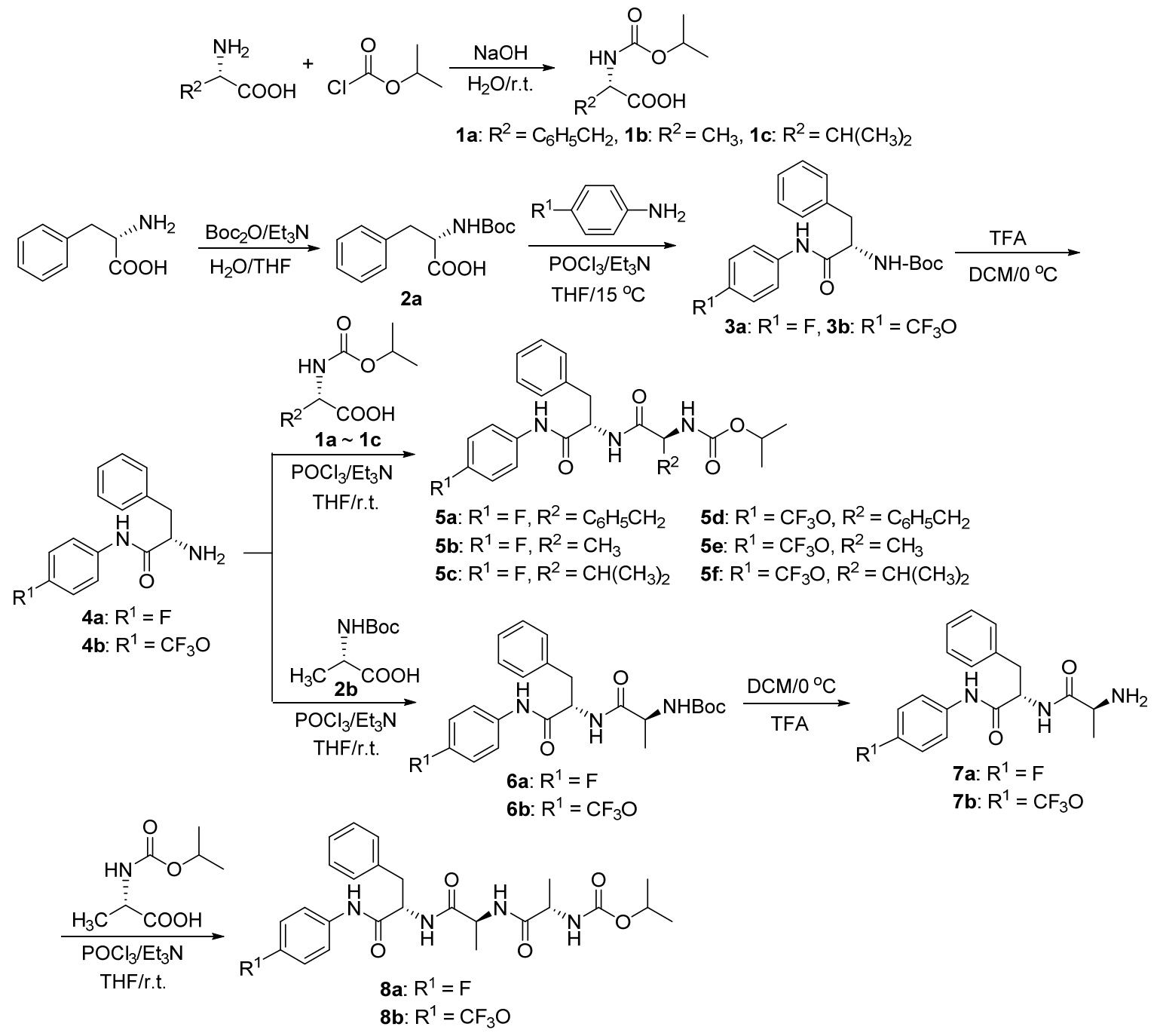

图式 1 苯丙氨酸衍生物的小分子肽合成路线

Scheme 1 Synthetic route of the small peptides of phenylalanine derivatives

${ }^{13} \mathrm{C}$ NMR 核磁共振谱图中, 脂肪碳 $\delta 9.51,18.44,18.53$, $22.49,37.88,48.78,50.37,55.26,67.44,119.34,155.99$, $170.48,172.65,173.02$; 芳环碳: $\delta 121.15,122.07,126.89$, $128.59,129.62,137.81,138.42,144.13$. 元素分析 Anal. calcd for $\mathrm{C}_{26} \mathrm{H}_{31} \mathrm{~N}_{4}$ : C 56.52, H 5.65, N 10.14; found C $56.54, \mathrm{H}$ 5.64, N 10.16. 说明产品纯度高. 高分辨质谱 HRMS calcd for $\mathrm{C}_{26} \mathrm{H}_{31} \mathrm{~F}_{3} \mathrm{~N}_{4} \mathrm{NaO}_{6}[\mathrm{M}+\mathrm{Na}]^{+}$575.2098, $[2 \mathrm{M}+\mathrm{Na}]^{+}$1127.4268, found $[\mathrm{M}+\mathrm{Na}]^{+}$575.2094, $[2 \mathrm{M}+\mathrm{Na}]^{+} 1127.4259$. 说明分子量正确. 综合 FTIR, ${ }^{1} \mathrm{H}$ NMR、 ${ }^{13} \mathrm{C} N M R$ 、元素分析、HRMS 等数据分析, 充分 说明产品结构的正确性.

\section{3 抗肿瘤活性}

本文采用经典的溴化噻唑蓝四氮唑法(MTT)比色 法, 以喜树碱(camptothecin)作为阳性对照物, 对目标化 合物进行了体外抗肿瘤活性测试, 选用人肝癌细胞 $\mathrm{SMC} 7721$, 目标化合物 5a $\sim \mathbf{5 d}$ 和 8a, 8b 对肿瘤细胞增
殖的抑制作用如表 1 所示.

表 1 目标化合物对肝癌细胞 SMC7721 增殖的抑制率(\%)

Table 1 The inhibition rate (\%) of the target compounds to hepatoma SMC7721 cells proliferation

\begin{tabular}{ccccc}
\hline \multirow{2}{*}{ Compound } & \multicolumn{4}{c}{ Concentration $/\left(\mu \mathrm{mol} \cdot \mathrm{mL}^{-1}\right)$} \\
\cline { 2 - 5 } & 0.5 & 1 & 2 & 4 \\
\hline $\mathbf{5 a}$ & 3.94 & 14.66 & 21.99 & 35.15 \\
$\mathbf{5 b}$ & 43.81 & 53.01 & 62.02 & 66.52 \\
$\mathbf{5 c}$ & 21.15 & 34.40 & 48.37 & 54.61 \\
$\mathbf{5 d}$ & 16.34 & 17.01 & 26.62 & 40.64 \\
$\mathbf{5 e}$ & 47.66 & 61.11 & 73.83 & 82.17 \\
$\mathbf{5 f}$ & 23.21 & 47.54 & 63.21 & 73.90 \\
$\mathbf{8 a}$ & 24.43 & 33.59 & 39.27 & 55.59 \\
$\mathbf{8 b}$ & 63.56 & 78.03 & 81.12 & 82.29 \\
Camptothecin & 81.04 & 83.92 & 84.47 & 86.79 \\
\hline
\end{tabular}

从表 1 的测试结果可以看出: (1)所有目标化合物在 $2 \mu \mathrm{mol} / \mathrm{mL}$ 时对肝癌细胞 SMC7721 的增殖表现出较好 的抑制效果，并且随着浓度的增加抑制效果也变得更加 
明显. (2)在各浓度中 4-三氟甲氧基苯胺系列要比对应的 4-氟苯胺系列化合物的抗肿瘤活性高, 推其原因可能是 与化合物作用靶点的电子云密度有关. (3) 4-三氟甲氧基 苯胺系列化合物中的三肽又较二肽的抗肿瘤活性要好, 说明在本系列中增加氨基酸的引入可以提高化合物的 抗肿瘤活性. 此外, 我们发现在高浓度时 $5 \mathrm{e}$ 和 $8 \mathrm{~b}$ 与喜 树碱对肝癌细胞 SMC7721 的抑制率接近, 可作为潜在 的抗肿瘤药物.

\section{2 结论}

本文以 $L$-苯丙氨酸和 4-氟苯胺(4-三氟甲氧基苯胺) 为起始原料, 经氨基保护、酰胺缩合、脱保护、再缩合、 再脱保护、再缩合等多步反应, 设计并合成了一系列 8 个未被文献报道的苯丙氨酸衍生物的小分子肽. 通过本 文合成路线获得的产品收率较高、纯度较好, 并且反应 条件温和, 操作简单, 后处理便捷. 在肽键合成中, 我 们用三氯氧磷代替昂贵的传统缩合剂, 从单个氨基酸的 缩合到多个氨基酸的缩合均只用三氯氧磷一种缩合剂 即可, 降低了成本、提高了产率、简化了后处理的操作 步骤. 同时研究了反应时间、反应温度、物料比等因素 对各步反应的影响, 全程通过 HPLC 跟踪监测, 确定了 最佳反应条件, 并采用 FTIR、 ${ }^{1} \mathrm{H}$ NMR 、 ${ }^{13} \mathrm{C}$ NMR、HRMS 和元素分析等测试方法对目标化合物的结构进行确认. 此外, 本文还对所有目标化合物进行了抗肿瘤活性测 试. 测试结果表明, 所有目标化合物在 $2 \mu \mathrm{mol} / \mathrm{mL}$ 时对 肝癌细胞 SMC7721 的增殖表现出较好的抑制效果, 其 中 $5 \mathrm{e}$ 和 $8 \mathrm{~b}$ 对肝癌细胞抑制效果较强, 与喜树碱对肝癌 细胞抑制效果相近, 可作为抗肿瘤药物先导化合物, 对 其结构进行更加深入的研究, 为新型抗肿瘤药物的开发 提供一定的理论依据.

\section{3 实验部分}

\section{1 仪器与试剂}

熔点采用 MPA-100 全自动熔点仪测定(温度计未校 正); 比旋光度采用上海申光 WXG-4 旋光仪测定; ${ }^{1} \mathrm{H}$ $\mathrm{NMR}$ 和 ${ }^{13} \mathrm{C}$ NMR 采用 Bruker-400 型核磁共振仪测定(以 $\mathrm{CDCl}_{3}$ 或 DMSO- $d_{6}$ 做溶剂), 以 TMS 为内标; FTIR 采用 FTIR-8400S 型红外光谱仪测定; ESI-HRMS 采用 micrOTOF-Q II 四极杆-飞行时间质谱仪测定; 元素分析 采用 Yanaco MT-3CHN 测定. 喜树碱(camptothecin)对照 物(上海晶纯生化科技股份有限公司提供), 其它所用试 剂均为分析纯或化学纯试剂, 所有氨基酸均为 $L$ 型, 四 氢呋喃、二氯甲烷用之前经干燥处理.

各化合物纯度均由高压液相色谱仪 LC-20A 测试结 果为依据, 测试方法: 采用面积归一化法; HPLC 分析条
件: Spursil C18-EP 色谱柱，波长 $254 \mathrm{~nm}$, 流动相为 $V($ 乙 腈) $: V$ (二次蒸馏水 $)=70: 30$, 流速 $0.8 \mathrm{~mL} / \mathrm{min}$.

\section{2 化合物的制备}

\section{2 .1 化合物 $\mathbf{1 a} \sim \mathbf{1 c}$ 的合成}

以 $1 \mathrm{a}$ 为例: $500 \mathrm{~mL}$ 装有搅拌器、温度计、空气冷 凝管和滴液漏斗的四口瓶中, 加入 $240 \mathrm{~mL}$ 水和 $16.8 \mathrm{~g}$ $(0.42 \mathrm{~mol})$ 氢氧化钠, 搅拌溶解, 再加入 $66.1 \mathrm{~g}(0.4 \mathrm{~mol})$ $L$-苯丙氨酸, 冰浴条件下, 搅拌滴加 $51.5 \mathrm{~g}(0.42 \mathrm{~mol})$ 氯 甲酸异丙酯, 控制温度小于 $5{ }^{\circ} \mathrm{C}$, 滴加完毕后, 升至室 温继续反应, HPLC 跟踪监测, $4 \mathrm{~h}$ 反应完毕, 冰水冷却, 用 $10 \%$ 的盐酸调节 $\mathrm{pH}$ 为 $2 \sim 3$, 用乙酸乙酯萃取 3 次, 合并有机层并用饱和食盐水洗涤, 无水硫酸镁干燥, 最 后浓缩旋出溶剂, 得到化合物 $\mathbf{1 a}$.

$L$ - 苯丙氨酰异丙酯 (1a): 白色膏状固体, 纯度 97.8\%, 收率 93.3\%. ${ }^{1} \mathrm{H}$ NMR (400 MHz, DMSO- $\left.d_{6}\right) \delta$ : $1.06(\mathrm{~d}, J=6.2 \mathrm{~Hz}, 3 \mathrm{H}), 1.14(\mathrm{~d}, J=6.2 \mathrm{~Hz}, 3 \mathrm{H}), 2.80 \sim$ $2.86(\mathrm{~m}, 1 \mathrm{H}), 3.02 \sim 3.07(\mathrm{~m}, 1 \mathrm{H}), 4.12 \sim 4.17(\mathrm{~m}, 1 \mathrm{H})$, $4.62 \sim 4.69(\mathrm{~m}, 1 \mathrm{H}), 7.19 \sim 7.34(\mathrm{~m}, 6 \mathrm{H}), 12.32$ (brs, $1 \mathrm{H}$, $\mathrm{COOH})$.

$L$-丙氨酰异丙酯( 以 $L$-丙氨酸为原料) $(\mathbf{1 b})$ : 淡黄色 油状物, 纯度 97.1\%, 收率 94.5\%. ${ }^{1} \mathrm{H}$ NMR (400 MHz, $\left.\mathrm{CDCl}_{3}\right) \delta: 1.23(\mathrm{~d}, J=6.2 \mathrm{~Hz}, 3 \mathrm{H}), 1.45(\mathrm{~d}, J=7.2 \mathrm{~Hz}$, $3 \mathrm{H}), 4.27 \sim 4.44(\mathrm{~m}, 1 \mathrm{H}), 4.84 \sim 4.92(\mathrm{~m}, 1 \mathrm{H}), 6.83$ (brs, 1H, NH), 9.99 (brs, 1H, COOH).

$L$-缴氨酰异丙酯(以 $L$-缬氨酸为原料) $(1 \mathrm{c})$ : 淡黄色 油状物，纯度 97.1\%，收率 93.6\%. ${ }^{1} \mathrm{H}$ NMR (400 MHz, DMSO- $\left.d_{6}\right) \delta: 0.86(\mathrm{~d}, J=6.8 \mathrm{~Hz}, 6 \mathrm{H}), 1.14(\mathrm{~d}, J=3.6 \mathrm{~Hz}$, $6 \mathrm{H}), 1.97 \sim 2.06(\mathrm{~m}, 1 \mathrm{H}), 3.82 \sim 3.87(\mathrm{~m}, 1 \mathrm{H}), 4.70 \sim 4.77$ (m, 1H), 7.06 (brs, 1H, NH).

\section{2 .2 化合物 $\mathbf{2 a}$ 和 $\mathbf{2 b}$ 的合成}

以 $\mathbf{2 a}$ 为例: $1000 \mathrm{~mL}$ 装有搅拌器、温度计、滴液漏 斗和空气冷凝管的四口瓶中, 加入 $200 \mathrm{~mL}$ 水和 $200 \mathrm{~mL}$ 四氢呋喃, 搅拌, 加入 $101.2 \mathrm{~g}(1 \mathrm{~mol})$ 三乙胺, 再加入 $82.5 \mathrm{~g}(0.5 \mathrm{~mol}) L$-苯丙氨酸, 冰浴条件下, 搅拌滴加 $109.1 \mathrm{~g}$ (0.5 mol) Boc 酸酐(溶于 $200 \mathrm{~mL}$ 四氢呋喃中), 滴毕撤去冰浴拌, 升温至 $30{ }^{\circ} \mathrm{C}$, HPLC 跟踪监测, 反应 $5 \mathrm{~h}$ 完毕, 减压蒸出溶剂, 冷却, 用饱和柠檬酸溶液调 $\mathrm{pH}$ 为 $2 \sim 3$, 用二氯甲烷萃取 3 次, 合并有机层并用饱和 食盐水洗涤, 无水硫酸镁干燥, 最后浓缩旋出溶剂, 得 到化合物 $\mathbf{2 a}$.

Boc- $L$-苯丙氨酸 $(2 a)$ : 白色固体, 纯度 $98.1 \%$, 收率 94.2\%. m.p. 84.3 84.8 ${ }^{\circ} \mathrm{C}$ (文献值 $84.0 \sim 86.0{ }^{\circ} \mathrm{C}$ ); ${ }^{1} \mathrm{H}$ NMR (400 MHz, DMSO- $\left.d_{6}\right) \delta: 1.32(\mathrm{~s}, 9 \mathrm{H}), 2.81 \sim 2.87$ $(\mathrm{m}, 1 \mathrm{H}), 3.01 \sim 3.05(\mathrm{~m}, 1 \mathrm{H}), 4.09 \sim 4.15(\mathrm{~m}, 1 \mathrm{H}), 7.07(\mathrm{~d}$, $J=8.4 \mathrm{~Hz}, 1 \mathrm{H}, \mathrm{NH}), 7.19 \sim 7.29$ (m, 5H), 12.62 (brs, 1H, 
$\mathrm{COOH})$.

Boc- $L$-丙氨酸(以 $L$-丙氨酸为原料) (2b): 白色固体, 纯度 $97.8 \%$, 收率 93.1\%. m.p. 82.2 82.8 ${ }^{\circ} \mathrm{C}$ (文献值 $\left.80.0 \sim 83.0{ }^{\circ} \mathrm{C}\right) ;{ }^{1} \mathrm{H}$ NMR (400 MHz, DMSO- $\left.d_{6}\right) \delta: 1.21$ $(\mathrm{d}, J=7.4 \mathrm{~Hz}, 3 \mathrm{H}), 1.36(\mathrm{~s}, 9 \mathrm{H}), 3.88 \sim 3.95(\mathrm{~m}, 1 \mathrm{H}), 7.04$ (d, $J=7.6 \mathrm{~Hz}, 1 \mathrm{H}, \mathrm{NH}), 12.33$ (brs, $1 \mathrm{H}, \mathrm{COOH}$ ).

\section{2 .3 化合物 $\mathbf{3 a}$ 和 $\mathbf{3 b}$ 的合成}

以 3a 为例: $1000 \mathrm{~mL}$ 装有搅拌器、温度计、滴液漏 斗和空气冷凝管的四口瓶中, 加入 $200 \mathrm{~mL}$ 无水四氢呋 喃, 搅拌下加入 $22.2 \mathrm{~g}(0.2 \mathrm{~mol})$ 对氟苯胺, 再加入 $58.3 \mathrm{~g}$ $(0.22 \mathrm{~mol})$ 化合物 $2 \mathbf{a}$ 和 $60.7 \mathrm{~g}(0.6 \mathrm{~mol})$ 三乙胺, 冰盐浴 条件下, 滴加 $30.7 \mathrm{~g}(0.2 \mathrm{~mol})$ 三氯氧磷, 控制温度在 $0{ }^{\circ} \mathrm{C}$ 以下, 滴毕, 控制 $10 \sim 15{ }^{\circ} \mathrm{C}$ 反应, HPLC 跟踪监测, $2 \mathrm{~h}$ 反应完毕, 过滤, 并用乙酸乙酯冲洗滤饼, 然后将有 机层用饱和 $\mathrm{NaHCO}_{3}$ 溶液洗 1 次, 饱和食盐水洗 2 次, 无 水硫酸镁干燥, 减压旋出溶剂, 粗产品用乙醚重结晶, 得到化合物 $\mathbf{3 a}$.

4-氟苯胺- $L$-苯丙氨酸-Boc (3a): 白色粉末固体, 纯 度 $98.5 \%$, 收率 $85.5 \%$. m.p. $168.8 \sim 169.2{ }^{\circ} \mathrm{C} ;[\alpha]_{\mathrm{D}}^{20}+$ 29.8 (c 1.0, DMF); ${ }^{1} \mathrm{H}$ NMR (400 MHz, DMSO- $d_{6}$ ) $\delta: 1.31$ $(\mathrm{s}, 9 \mathrm{H}), 2.81 \sim 2.89(\mathrm{~m}, 1 \mathrm{H}), 2.96 \sim 3.01(\mathrm{~m}, 1 \mathrm{H}), 4.28 \sim$ $4.34(\mathrm{~m}, 1 \mathrm{H}), 7.09 \sim 7.32(\mathrm{~m}, 8 \mathrm{H}), 7.60(\mathrm{dd}, J=8.9,5.0$ $\mathrm{Hz}, 2 \mathrm{H}), 10.14(\mathrm{~s}, 1 \mathrm{H}, \mathrm{NH}) ;{ }^{13} \mathrm{C} \mathrm{NMR}(100 \mathrm{MHz}$, DMSO- $\left.d_{6}\right) \delta: 171.21,159.69,157.30,155.88,138.42$, $135.82,129.74,128.51,126.77,121.57,121.49,115.84$, $115.62,78.57,57.04,45.85,37.95,28.63,9.49$.

4-三氟甲氧基苯胺- $L$-苯丙氨酸-Boc(以 4-三氟甲氧 基苯胺为原料)(3b): 白色粉末固体, 纯度 $98.7 \%$, 收率 85.7\%. m.p. $122.6 \sim 123.1{ }^{\circ} \mathrm{C} ;[\alpha]_{\mathrm{D}}^{20}+40.7$ (c 1.0, DMF); ${ }^{1} \mathrm{H}$ NMR (400 MHz, DMSO- $\left.d_{6}\right) \delta: 1.32$ (s, 9H), $2.82 \sim 2.88(\mathrm{~m}, 1 \mathrm{H}), 2.96 \sim 3.01(\mathrm{~m}, 1 \mathrm{H}), 4.29 \sim 4.35(\mathrm{~m}$, $1 \mathrm{H}), 7.16 \sim 7.38(\mathrm{~m}, 8 \mathrm{H}), 7.63(\mathrm{~d}, J=8.9 \mathrm{~Hz}, 2 \mathrm{H}), 10.20$ $(\mathrm{s}, 1 \mathrm{H}, \mathrm{NH}) ;{ }^{\mathrm{I}} \mathrm{C}$ NMR $\left(100 \mathrm{MHz}, \mathrm{DMSO}-d_{6}\right) \delta: 171.62$, $155.92,144.05,138.72,138.39$, 129.77, 128.52, 126.79, $122.07,121.10,119.37,78.60,57.15,45.79,37.83,28.63$, 9.07 .

\section{2 .4 化合物 $4 \mathrm{a}$ 和 $4 \mathrm{~b}$ 的合成}

以 $4 \mathrm{a}$ 为例: $500 \mathrm{~mL}$ 装有搅拌器、温度计、空气冷 凝管的四口瓶中, 加入 $160 \mathrm{~mL}$ 二氯甲烷, $68.4 \mathrm{~g}(0.6$

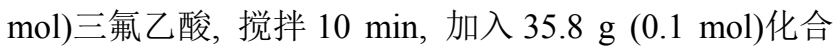
物 3a, 控制 $0{ }^{\circ} \mathrm{C}$ 反应, HPLC 跟踪监测, 搅拌反应 $4 \mathrm{~h}$, 减压蒸出二氯甲烷和过量的三氟乙酸, 冷却, 用饱和碳 酸氢钠溶液调 $\mathrm{pH}$ 为 $7 \sim 8$, 用二氯甲烷萃取三次, 合并 有机层并用饱和食盐水洗涤, 无水硫酸镁干燥, 最后浓 缩旋出溶剂, 粗产品用乙醚重结晶, 得到化合物 $4 \mathbf{a}$.
4-氟苯胺- $L$-苯丙氨酸 $(4 \mathbf{a})$ : 白色粉末固体, 纯度 97.7\%, 收率 $86.5 \%$. m.p. $189.4 \sim 190.1{ }^{\circ} \mathrm{C}$; $[\alpha]_{\mathrm{D}}^{20}+19.9$ (c 1.0, DMF); ${ }^{1} \mathrm{H}$ NMR (400 MHz, DMSO- $\left.d_{6}\right) \delta: 3.07 \sim$ $3.19(\mathrm{~m}, 2 \mathrm{H}), 4.16(\mathrm{t}, J=7.0 \mathrm{~Hz}, 1 \mathrm{H}), 7.16 \sim 7.34(\mathrm{~m}, 7 \mathrm{H})$, $7.52(\mathrm{dd}, J=9.1,5.0 \mathrm{~Hz}, 2 \mathrm{H}), 8.35\left(\mathrm{~s}, 2 \mathrm{H}, \mathrm{NH}_{2}\right), 10.53$ (s, $1 \mathrm{H}, \mathrm{NH}) ;{ }^{13} \mathrm{C}$ NMR $\left(100 \mathrm{MHz}, \mathrm{DMSO}-d_{6}\right) \delta: 167.26$, $160.21,159.23,158.91,157.82,135.36,134.86,130.00$, $129.05,127.72,122.08,122.00,119.24,116.15,115.93$, $54.77,37.60$.

4-三氟甲氧基苯胺- $L$-苯丙氨酸(以 $3 b$ 为原料)(4b): 白色粉末固体，纯度 $98.3 \%$, 收率 86.6\%. m.p. 106.3 $106.9{ }^{\circ} \mathrm{C} ;[\alpha]_{\mathrm{D}}^{20}+24.7$ (c 1.0, DMF); ${ }^{1} \mathrm{H}$ NMR $(400$ MHz, DMSO- $\left.d_{6}\right) \delta: 2.72 \sim 2.77(\mathrm{~m}, 1 \mathrm{H}), 2.99 \sim 3.03(\mathrm{~m}$, $1 \mathrm{H}), 3.55 \sim 3.61(\mathrm{~m}, 1 \mathrm{H}), 7.19 \sim 7.31(\mathrm{~m}, 7 \mathrm{H}), 7.72(\mathrm{~d}, J=$ $9.1 \mathrm{~Hz}, 2 \mathrm{H}) ;{ }^{13} \mathrm{C}$ NMR (100 MHz, DMSO- $\left.d_{6}\right) \delta: 174.18$, $144.05,138.97,138.55,129.78,128.61,126.66,122.03$, $121.10,119.37,57.59,41.42$.

\section{2 .5 化合物 $5 \mathbf{a} \sim 5 \mathrm{ff}$ 和 $\mathbf{6 a} \sim \mathbf{6 b}$ 的合成}

以 $5 \mathbf{a}$ 为例: $100 \mathrm{~mL}$ 装有搅拌器、温度计、空气冷 凝管和滴液漏斗的四口瓶中, 加入 $60 \mathrm{~mL}$ 无水四氢呋 喃, 搅拌下加入 $2.58 \mathrm{~g}(0.01 \mathrm{~mol})$ 化合物 $\mathbf{4 a}$, 再加入 2.76 $\mathrm{g}(0.011 \mathrm{~mol})$ 化合物 $1 \mathrm{a}$ 和 $3.54 \mathrm{~g}(0.035 \mathrm{~mol})$ 三乙胺, 冰 盐浴条件下, 滴加 $1.53 \mathrm{~g}(0.01 \mathrm{~mol})$ 三氯氧磷, 控制温度 在 $0{ }^{\circ} \mathrm{C}$ 以下, HPLC 监测反应, 室温 $2 \mathrm{~h}$ 反应完毕, 过滤, 并将滤饼用乙酸乙酯冲洗，合并有机层用 $20 \mathrm{~mL}$ 饱和 $\mathrm{NaHCO}_{3}$ 溶液洗 1 次, $50 \mathrm{~mL}$ 饱和食盐水洗 2 次, 无水硫 酸镁干燥, 减压浓缩得到固体粗产品, 向蒸馏瓶中加入 乙醚/甲醇 $[V($ 乙醚 $) / V$ (甲醇 $)=20 / 1]$, 对粗产品进行重结 晶, 冷却得到化合物 $\mathbf{5 a}$.

4-氟苯胺- $L$-苯丙氨酰- $L$-苯丙氨酰异丙酯 $(\mathbf{5 a})$ : 白色 粉末固体，纯度 $98.3 \%$, 收率 84.6\%. m.p. 231.7 $232.2{ }^{\circ} \mathrm{C} ;[\alpha]_{\mathrm{D}}^{20}+12.6$ (c 1.0, DMF); ${ }^{1} \mathrm{H}$ NMR $(400$ $\left.\mathrm{MHz}, \mathrm{DMSO}-d_{6}\right) \delta$ : $1.04(\mathrm{~d}, J=6.0 \mathrm{~Hz}, 3 \mathrm{H}), 1.11(\mathrm{~d}, J=$ $6.0 \mathrm{~Hz}, 3 \mathrm{H}), 2.64 \sim 2.70(\mathrm{~m}, 1 \mathrm{H}), 2.89 \sim 2.97(\mathrm{~m}, 2 \mathrm{H})$, $3.04 \sim 3.09(\mathrm{~m}, 1 \mathrm{H}), 4.18 \sim 4.23(\mathrm{~m}, 1 \mathrm{H}), 4.61 \sim .67(\mathrm{~m}$, $2 \mathrm{H}), 7.13 \sim 7.30(\mathrm{~m}, 13 \mathrm{H}), 7.57(\mathrm{~d}, J=8.1 \mathrm{~Hz}, 2 \mathrm{H}), 8.25$ $(\mathrm{d}, J=7.4 \mathrm{~Hz}, 1 \mathrm{H}, \mathrm{NH}), 10.11(\mathrm{~s}, 1 \mathrm{H}, \mathrm{NH}) ;{ }^{13} \mathrm{C} \mathrm{NMR}(100$ $\left.\mathrm{MHz}, \mathrm{DMSO}-d_{6}\right) \delta$ : 171.99, 170.16, 159.77, 157.39, $156.01,138.44,137.79,136.56,129.72,129.64,128.60$, $128.43,126.91,126.64,121.63,121.55,115.87,115.65$, 67.46, 56.50, 55.17, 38.27, 37.95, 22.40; IR (KBr) v: 3299, 3275, 2980, 1689, 1652, 1532, 1510, 1289, 1219, 832, 699 $\mathrm{cm}^{-1}$. Anal. calcd for $\mathrm{C}_{28} \mathrm{H}_{30} \mathrm{~N}_{4}$ : C 68.42, H 6.15, N 8.55; found C 68.45, H 6.13, N 8.57.

4 -氟苯胺- $L$-苯丙氨酰- $L$-丙氨酰异丙酯(以 $4 \mathbf{a}$ 和 $1 \mathbf{b}$ 
为原料)(5b): 白色固体, 纯度 $96.7 \%$, 收率 $80.2 \%$. m.p. $243.8 \sim 244.0{ }^{\circ} \mathrm{C}$; $[\alpha]_{\mathrm{D}}^{20}+12.5$ (c 1.0, DMF); ${ }^{1} \mathrm{H}$ NMR (400 MHz, DMSO-d $)_{6} \delta: 0.97$ (d, $\left.J=7.1 \mathrm{~Hz}, 3 \mathrm{H}\right), 1.14$ $(\mathrm{dd}, J=11.2,6.2 \mathrm{~Hz}, 6 \mathrm{H}), 2.83 \sim 2.88(\mathrm{~m}, 1 \mathrm{H}), 3.10 \sim 3.15$ $(\mathrm{m}, 1 \mathrm{H}), 3.94 \sim 4.07(\mathrm{~m}, 1 \mathrm{H}), 4.60 \sim 4.75(\mathrm{~m}, 2 \mathrm{H}), 7.14 \sim$ $7.27(\mathrm{~m}, 8 \mathrm{H}), 7.65(\mathrm{dd}, J=8.8,5.0 \mathrm{~Hz}, 2 \mathrm{H}), 8.32$ (d, $J=$ $8.4 \mathrm{~Hz}, 1 \mathrm{H}, \mathrm{NH}), 9.99$ (s, 1H, NH); ${ }^{13} \mathrm{C} \mathrm{NMR}(100 \mathrm{MHz}$, DMSO- $\left.d_{6}\right) \delta: 173.06,170.20,159.77,157.38,156.02$, $137.88,135.59,129.71,128.54,126.85,121.55,121.51$, $115.87,115.65,67.56,55.00,50.64,38.08,22.50$, 18.50; IR (KBr) v: 3292, 2981, 1689, 1653, 1536, 1511, 1220, $834,699 \mathrm{~cm}^{-1}$. Anal. calcd for $\mathrm{C}_{22} \mathrm{H}_{26} \mathrm{~N}_{3}$ : C 63.60, H 6.31, N 10.11; found C 63.62, H 6.30, N 10.12.

4-氟苯胺- $L$-苯丙氨酰- $L$-巅氨酰异丙酯(以 $4 \mathbf{a}$ 和 $1 \mathrm{c}$ 为原料)(5c): 白色固体, 纯度 $97.1 \%$, 收率 79.2\%. m.p. $209.3 \sim 209.9{ }^{\circ} \mathrm{C} ;[\alpha]_{\mathrm{D}}^{20}+14.9$ (c 1.0, DMF); ${ }^{1} \mathrm{H}$ NMR $\left(400 \mathrm{MHz}, \mathrm{DMSO}-d_{6}\right) \delta: 0.73(\mathrm{t}, J=7.5 \mathrm{~Hz}, 6 \mathrm{H}), 1.16(\mathrm{t}$, $J=5.5 \mathrm{~Hz}, 6 \mathrm{H}), 1.84 \sim 1.89(\mathrm{~m}, 1 \mathrm{H}), 2.87 \sim 2.93(\mathrm{~m}, 1 \mathrm{H})$, $3.02 \sim 3.07(\mathrm{~m}, 1 \mathrm{H}), 3.77 \sim 3.82(\mathrm{~m}, 1 \mathrm{H}), 4.65 \sim 4.76(\mathrm{~m}$, $2 \mathrm{H}), 6.94(\mathrm{~d}, J=8.8 \mathrm{~Hz}, 1 \mathrm{H}, \mathrm{NH}), 7.11 \sim 7.28(\mathrm{~m}, 7 \mathrm{H})$, $7.56(\mathrm{dd}, J=9.0,5.0 \mathrm{~Hz}, 2 \mathrm{H}), 8.11(\mathrm{~d}, J=8.0 \mathrm{~Hz}, 1 \mathrm{H}$, $\mathrm{NH}), 10.07$ (s, 1H, NH); ${ }^{13} \mathrm{C}$ NMR (100 MHz, DMSO- $\left.d_{6}\right)$ $\delta: 171.65,170.24,159.74,157.35,156.32,137.85,135.56$, $126.83,121.47,115.85,115.63,67.55,60.70,55.00,38.17$, 30.83, 22.46, 19.52, 18.59; IR (KBr) v: 3290, 2975, 1691, $1648,1540,1511,1222,833,700 \mathrm{~cm}^{-1}$. Anal. calcd for $\mathrm{C}_{28} \mathrm{H}_{30} \mathrm{~N}_{3}$ : C 68.42, H 6.15, N 8.55; found C 68.45, H 6.11, N 8.58.

4-三氟甲氧基苯胺- $L$-苯丙氨酰- $L$-苯丙氨酰异丙酯 (以 $4 b$ 和 $1 \mathrm{a}$ 为原料)(5d): 白色粉末固体，纯度 $98.7 \%$, 收率 $83.0 \%$. m.p. $203.2 \sim 203.9{ }^{\circ} \mathrm{C}$; $[\alpha]_{\mathrm{D}}^{20}+15.0$ (c 1.0, DMF); ${ }^{1} \mathrm{H}$ NMR (400 MHz, DMSO- $\left.d_{6}\right) \delta: 1.04$ (d, $J=6.2$ $\mathrm{Hz}, 3 \mathrm{H}), 1.11(\mathrm{~d}, J=6.2 \mathrm{~Hz}, 3 \mathrm{H}), 2.64 \sim 2.70(\mathrm{~m}, 1 \mathrm{H})$, $2.88 \sim 2.97(\mathrm{~m}, 2 \mathrm{H}), 3.05 \sim 3.10(\mathrm{~m}, 1 \mathrm{H}), 4.19 \sim 4.24(\mathrm{~m}$, $1 \mathrm{H}), 4.59 \sim 4.71(\mathrm{~m}, 2 \mathrm{H}), 7.13(\mathrm{~d}, J=8.6 \mathrm{~Hz}, 1 \mathrm{H}, \mathrm{NH})$, $7.20 \sim 7.33(\mathrm{~m}, 12 \mathrm{H}), 7.67(\mathrm{~d}, J=9.0 \mathrm{~Hz}, 2 \mathrm{H}), 8.30(\mathrm{~d}, \mathrm{~J}=$ $7.8 \mathrm{~Hz}, 1 \mathrm{H}, \mathrm{NH}), 10.28$ (s, 1H, NH); ${ }^{\mathrm{I}} \mathrm{C} \mathrm{NMR}(100 \mathrm{MHz}$, DMSO- $\left.d_{6}\right) \delta: 172.09,170.54,156.03,144.18,138.46$, $138.40,137.75,129.72,129.65,128.63,128.44,126.95$, $126.64,122.11,121.16,119.35,67.46,56.48,55.27,38.17$, 37.96, 22.41; IR (KBr) v: 3282, 2980, 1689, 1651, 1533, $1280,1243,1223,699 \mathrm{~cm}^{-1}$. Anal. calcd for $\mathrm{C}_{29} \mathrm{H}_{30} \mathrm{~N}_{3}: \mathrm{C}$ 62.47, H 5.42, N 7.54; found C 62.48, H 5.40, N 7.56.

4-三氟甲氧基苯胺- $L$-苯丙氨酰- $L$-丙氨酰异丙酯(以 4b 和 $1 \mathrm{~b}$ 为原料)(5e): 白色固体, 纯度 $99.1 \%$, 收率
81.1\%. m.p. $213.8 \sim 214.3{ }^{\circ} \mathrm{C} ;[\alpha]_{\mathrm{D}}^{20}+15.1$ (c 1.0 , DMF); ${ }^{1} \mathrm{H}$ NMR (400 MHz, DMSO- $\left.d_{6}\right) \delta: 1.14$ (brs, 9H), $2.85 \sim 3.00(\mathrm{~m}, 1 \mathrm{H}), 3.08(\mathrm{~d}, J=9.7 \mathrm{~Hz}, 1 \mathrm{H}), 4.01$ (brs, $1 \mathrm{H}), 4.66 \sim 4.73(\mathrm{~m}, 2 \mathrm{H}), 7.18 \sim 7.31(\mathrm{~m}, 8 \mathrm{H}), 7.68(\mathrm{~d}, J=$ $7.9 \mathrm{~Hz}, 2 \mathrm{H}), 8.08$ (d, J=7.1 Hz, 1H, NH), 10.17 (s, 1H, $\mathrm{NH}) ;{ }^{13} \mathrm{C}$ NMR (100 MHz, DMSO- $\left.d_{6}\right) \delta: 173.13,170.55$, $156.01,144.17,138.39,137.81,129.69,128.57,126.88$, $122.10,121.13,119.35,67.55,55.08,50.58,38.00,22.49$, 18.50; IR (KBr) v: 3292, 2983, 1686, 1649, 1539, 1510, 1264, 1220, 842, $700 \mathrm{~cm}^{-1}$. Anal. calcd for $\mathrm{C}_{23} \mathrm{H}_{26} \mathrm{~N}_{3}: \mathrm{C}$ 57.38, H 5.44, N 8.73; found $\mathrm{C} 57.40, \mathrm{H} 5.41, \mathrm{~N} 8.75$; HRMS calcd for $\mathrm{C}_{23} \mathrm{H}_{26} \mathrm{~F}_{3} \mathrm{~N}_{3} \mathrm{NaO}_{5}[\mathrm{M}+\mathrm{Na}]^{+}$504.1698, $[2 \mathrm{M}+\mathrm{Na}]^{+}$985.3528; found $[\mathrm{M}+\mathrm{Na}]^{+}$504.1718, $[2 \mathrm{M}+$ $\mathrm{Na}]^{+} 985.3547$.

4-三氟甲氧基苯胺- $L$-苯丙氨酰- $L$-倾氨酰异丙酯(以 4b 和 1c 为原料)(5f): 白色固体，纯度 $97.1 \%$, 收率 79.2\%. m.p. $209.3 \sim 209.9{ }^{\circ} \mathrm{C} ;[\alpha]_{\mathrm{D}}^{20}+15.6$ (c 1.0, DMF); ${ }^{1} \mathrm{H}$ NMR (400 MHz, DMSO- $\left.d_{6}\right) \delta: 0.73$ (t, $J=7.2$ $\mathrm{Hz}, 6 \mathrm{H}), 1.16(\mathrm{t}, J=5.8 \mathrm{~Hz}, 6 \mathrm{H}), 1.84 \sim 1.89(\mathrm{~m}, 1 \mathrm{H})$, $2.88 \sim 2.94(\mathrm{~m}, 1 \mathrm{H}), 3.03 \sim 3.08(\mathrm{~m}, 1 \mathrm{H}), 3.79 \sim 3.83(\mathrm{~m}$, $1 \mathrm{H}), 4.67 \sim 4.76(\mathrm{~m}, 2 \mathrm{H}), 6.94(\mathrm{~d}, J=8.8 \mathrm{~Hz}, 1 \mathrm{H}, \mathrm{NH})$, $7.17 \sim 7.32(\mathrm{~m}, 7 \mathrm{H}), 7.67(\mathrm{~d}, J=9.0 \mathrm{~Hz}, 2 \mathrm{H}), 8.17(\mathrm{~d}, J=$ $7.9 \mathrm{~Hz}, 1 \mathrm{H}, \mathrm{NH}), 10.27$ (s, 1H, NH); ${ }^{13} \mathrm{C}$ NMR (100 MHz, DMSO- $\left.d_{6}\right) \delta: 171.73,170.63,156.32,144.13,138.43$, $137.81,128.55,126.87,122.09,121.09,119.34,67.54$, $60.67,55.12,38.08,30.85,22.46,19.52$, 18.60; IR (KBr) $v: 3298,2978,1690,1649,1540,1511,1271,848,700$ $\mathrm{cm}^{-1}$. Anal. calcd for $\mathrm{C}_{29} \mathrm{H}_{30} \mathrm{~N}_{3}: \mathrm{C} 62.47, \mathrm{H} 5.42, \mathrm{~N} 7.54$; found C 62.51, H 5.40, N 7.57.

4-氟苯胺- $L$-苯丙氨酰- $L$-丙氨酸-Boc(以 $4 \mathbf{a}$ 和 $2 \mathbf{b}$ 为 原料)(6a): 白色粉末固体，纯度 $97.8 \%$, 收率 $83.9 \%$. m.p. $135.2 \sim 135.9{ }^{\circ} \mathrm{C} ;[\alpha]_{\mathrm{D}}^{20}+17.4$ (c 1.0, DMF); ${ }^{1} \mathrm{H}$ NMR (400 MHz, DMSO- $\left.d_{6}\right) \delta: 1.10(\mathrm{~d}, J=7.2 \mathrm{~Hz}, 3 \mathrm{H})$, $1.35(\mathrm{~s}, 9 \mathrm{H}), 2.90 \sim 2.96(\mathrm{~m}, 1 \mathrm{H}), 3.03 \sim 3.08(\mathrm{~m}, 1 \mathrm{H})$, $3.90 \sim 3.95(\mathrm{~m}, 1 \mathrm{H}), 4.62 \sim 4.67(\mathrm{~m}, 1 \mathrm{H}), 6.97(\mathrm{~d}, J=7.1$ $\mathrm{Hz}, 1 \mathrm{H}, \mathrm{NH}), 7.10 \sim 7.29(\mathrm{~m}, 7 \mathrm{H}), 7.59(\mathrm{dd}, J=8.8,5.0$ $\mathrm{Hz}, 2 \mathrm{H}), 8.05$ (d, $J=8.0 \mathrm{~Hz}, 1 \mathrm{H}, \mathrm{NH}), 10.17$ (s, 1H, NH); ${ }^{13} \mathrm{C}$ NMR (100 MHz, DMSO- $\left.d_{6}\right) \delta: 173.12,170.23,159.76$, $157.38,155.57,137.87,135.61,129.74,128.53,126.85$, $121.64,121.56,115.85,115.62,78.68,54.97,50.48,38.16$, 28.65, 18.55, 8.90; IR (KBr) v: 3299, 2980, 1690, 1660, $1541,1512,1219,1166,836,699 \mathrm{~cm}^{-1}$. Anal. calcd for $\mathrm{C}_{23} \mathrm{H}_{28} \mathrm{~N}_{3}$ : C 64.32, H 6.57, N 9.78; found C 64.35, H 6.54, N 9.74 .

4-三氟甲氧基苯胺- $L$-苯丙氨酰- $L$-丙氨酸- Boc(以 $4 b$ 
和 $2 \mathbf{b}$ 为原料)(6b): 白色粉末固体, 纯度 $97.7 \%$, 收率 80.5\%. m.p. $213.8 \sim 214.0{ }^{\circ} \mathrm{C} ;[\alpha]_{\mathrm{D}}^{20}+22.6$ (c 1.0, DMF); ${ }^{1} \mathrm{H}$ NMR (400 MHz, DMSO- $\left.d_{6}\right) \delta: 1.10$ (d, $J=7.2$ $\mathrm{Hz}, 3 \mathrm{H}), 1.35(\mathrm{~s}, 9 \mathrm{H}), 2.91 \sim 2.97(\mathrm{~m}, 1 \mathrm{H}), 3.04 \sim 3.09(\mathrm{~m}$, $1 \mathrm{H}), 3.88 \sim 4.01(\mathrm{~m}, 1 \mathrm{H}), 4.63 \sim 4.68(\mathrm{~m}, 1 \mathrm{H}), 6.96(\mathrm{~d}, J=$ $7.3 \mathrm{~Hz}, 1 \mathrm{H}, \mathrm{NH}), 7.18 \sim 7.32(\mathrm{~m}, 7 \mathrm{H}), 7.67(\mathrm{~d}, J=9.0 \mathrm{~Hz}$, $2 \mathrm{H}), 8.06(\mathrm{~d}, J=7.9 \mathrm{~Hz}, 1 \mathrm{H}, \mathrm{NH}), 10.28(\mathrm{~s}, 1 \mathrm{H}, \mathrm{NH}) ;{ }^{13} \mathrm{C}$ NMR (100 MHz, DMSO- $\left.d_{6}\right) \delta$ : 173.19, 170.57, 155.58, $144.17,138.41,137.79,129.72,128.56,126.88,122.08$, $121.17,119.35,78.66,55.03,50.42$, 45.89, 38.10, 28.64, 18.55, 9.55; IR (KBr) v: 3298, 2981, 1691, 1661, 1544, $1513,1270,1165,850,699 \mathrm{~cm}^{-1}$. Anal. calcd for $\mathrm{C}_{24} \mathrm{H}_{28}-$ $\mathrm{N}_{3}$ : C 58.18, H 5.70, N 8.48; found C 58.21, H 5.69, N 8.50 .

\section{2 .6 化合物 $7 \mathbf{a}$ 和 $7 \mathbf{b}$ 的合成}

以 7a 为例: $500 \mathrm{~mL}$ 装有搅拌器、温度计、空气冷 凝管的四口瓶中，加入 $168 \mathrm{~mL}$ 二氯甲烷，62.7 g (0.55

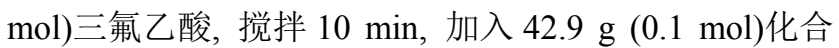
物 6a, 控制 $0{ }^{\circ} \mathrm{C}$, HPLC 跟踪反应, $40 \mathrm{~h}$ 反应完毕, 减压 蒸出二氯甲烷和过量的三氟乙酸, 冷却, 用饱和碳酸氢 钠溶液调 $\mathrm{pH}$ 为 $7 \sim 8$, 用二氯甲烷萃取 3 次, 合并有机 层用饱和食盐水洗涤, 无水硫酸镁干燥, 最后浓缩旋出 溶剂, 粗产品用乙醚重结晶, 得到化合物 7a.

4-氟苯胺- $L$-苯丙氨酰- $L$-丙氨酸 $(7 \mathbf{a})$ : 白色粉末固 体, 纯度 98.5\%, 收率 73.6\%. m.p. 193.7 194.2 ${ }^{\circ} \mathrm{C}$; $[\alpha]_{\mathrm{D}}^{20}+19.8$ (c 1.0, DMF); ${ }^{1} \mathrm{H}$ NMR (400 MHz, DMSO$\left.d_{6}\right) \delta: 1.02(\mathrm{~d}, J=6.9 \mathrm{~Hz}, 3 \mathrm{H}), 1.81$ (brs, $\left.2 \mathrm{H}, \mathrm{NH}_{2}\right), 2.89 \sim$ $2.95(\mathrm{~m}, 1 \mathrm{H}), 3.04 \sim 3.09(\mathrm{~m}, 1 \mathrm{H}), 3.21 \sim 3.26(\mathrm{~m}, 1 \mathrm{H})$, 4.67 (brs, $1 \mathrm{H}), 7.13 \sim 7.28(\mathrm{~m}, 7 \mathrm{H}), 7.58(\mathrm{dd}, J=9.0,5.0$ $\mathrm{Hz}, 2 \mathrm{H}), 8.14$ (brs, $1 \mathrm{H}, \mathrm{NH}), 10.16(\mathrm{~s}, 1 \mathrm{H}, \mathrm{NH}) ;{ }^{13} \mathrm{C} \mathrm{NMR}$ $\left(100 \mathrm{MHz}, \mathrm{DMSO}-d_{6}\right) \delta: 176.05,170.29,159.80,157.41$, $137.73,135.52,135.50,129.75,128.52,126.87,121.75$, $121.68,115.86,115.64,54.43,50.68,38.62,21.82$; IR $(\mathrm{KBr}) v: 3303,1650,1558,1509,1410,1217,834,700$ $\mathrm{cm}^{-1}$. Anal. calcd for $\mathrm{C}_{18} \mathrm{H}_{20} \mathrm{~N}_{3}$ : C 65.64, H 6.12, N 12.76; found C 65.68, H 6.09, N 12.73 .

4 -三氟甲氧基苯胺- $L$-苯丙氨酰- $L$-丙氨酸(以 $6 \mathbf{b}$ 为 原料)(7b): 白色粉末固体, 纯度 $98.1 \%$, 收率 $72.2 \%$. m.p. $170.9 \sim 171.3{ }^{\circ} \mathrm{C}$; $[\alpha]_{\mathrm{D}}^{20}+23.1$ (c 1.0, DMF); ${ }^{1} \mathrm{H}$ NMR (400 MHz, DMSO- $\left.d_{6}\right) \delta$ : $1.02(\mathrm{~d}, J=6.9 \mathrm{~Hz}, 3 \mathrm{H})$, 1.84 (brs, $\left.2 \mathrm{H}, \mathrm{NH}_{2}\right), 2.90 \sim 2.96(\mathrm{~m}, 1 \mathrm{H}), 3.04 \sim 3.09(\mathrm{~m}$, $1 \mathrm{H}), 3.22 \sim 3.27(\mathrm{~m}, 1 \mathrm{H}), 4.69$ (brs, $1 \mathrm{H}), 7.17 \sim 7.35(\mathrm{~m}$, $7 \mathrm{H}), 7.67$ (d, $J=9.0 \mathrm{~Hz}, 2 \mathrm{H}), 8.16$ (brs, 1H, NH), 10.32 (s, $1 \mathrm{H}, \mathrm{NH}) ;{ }^{13} \mathrm{C}$ NMR (100 MHz, DMSO- $\left.d_{6}\right) \delta: 176.11$, $170.65,144.18,138.36,137.68,129.75,128.55,126.90$,
$122.09,121.26,119.34,54.53,50.66,38.52,21.83$; IR (KBr) $v: 3319,3280,1690,1644,1554,1510,1269$, 1258, 1203, 1163, 843, $700 \mathrm{~cm}^{-1}$. Anal. calcd for $\mathrm{C}_{19} \mathrm{H}_{20} \mathrm{~N}_{3}$ : C 57.72, H 5.10, N 10.63; found C 57.75, H 5.07, N 10.60.

\subsection{7 化合物 $8 \mathrm{a}$ 和 $8 \mathrm{~b}$ 的合成}

以 $8 \mathbf{a}$ 为例: $100 \mathrm{~mL}$ 装有搅拌器、温度计、空气冷 凝管和滴液漏斗的四口瓶中, 加入 $50 \mathrm{~mL}$ 无水四氢呋 喃, 搅拌下加入 $1.65 \mathrm{~g}(0.005 \mathrm{~mol})$ 化合物 7a, 再加入 $1.05 \mathrm{~g}(0.006 \mathrm{~mol})$ 化合物 $\mathbf{1 b}$ 和 $2.53 \mathrm{~g}(0.025 \mathrm{~mol})$ 三乙胺, 冰盐浴条件下，滴加 $0.63 \mathrm{~g}(0.0055 \mathrm{~mol})$ 三氯氧磷，控制 温度在 $-10{ }^{\circ} \mathrm{C}$ 以下, 滴毕, 保持 $0{ }^{\circ} \mathrm{C}$ 以下反应, HPLC 跟踪监测, $2.5 \mathrm{~h}$ 后反应完毕, 过滤, 滤饼用乙酸乙酯冲 洗, 合并有机层用 $10 \mathrm{~mL}$ 饱和 $\mathrm{NaHCO}_{3}$ 溶液洗 1 次, 50 $\mathrm{mL}$ 饱和食盐水洗 2 次, 无水硫酸镁干燥, 减压浓缩得到 固体粗产品, 向蒸馏瓶中加入乙醚/甲醇 $[V($ 乙醚 $) / V($ 甲 醇 $)=10 / 1$, 对粗产品进行重结晶, 冷却得到化合物 $8 \mathbf{a}$.

4-氟苯胺- $L$ - 苯丙氨酰- $L$-丙氨酰- $L$-丙氨酰异丙酯 (以 $7 \mathbf{a}$ 和 $1 \mathbf{b}$ 为原料)(8a): 白色粉末固体, 纯度 $98.3 \%$, 收率 65.6\%. m.p. 231.4 231.9 ${ }^{\circ} \mathrm{C} ;[\alpha]_{\mathrm{D}}^{20}+9.9$ (c 1.0, DMF); ${ }^{1} \mathrm{H}$ NMR (400 MHz, DMSO- $\left.d_{6}\right) \delta: 1.15$ (t, $J=7.5$ $\mathrm{Hz}, 12 \mathrm{H}), 2.88 \sim 2.94(\mathrm{~m}, 1 \mathrm{H}), 3.04 \sim 3.09(\mathrm{~m}, 2 \mathrm{H}), 3.92 \sim$ $4.05(\mathrm{~m}, 1 \mathrm{H}), 4.12 \sim 4.41(\mathrm{~m}, 1 \mathrm{H}), 4.56 \sim 4.62(\mathrm{~m}, 1 \mathrm{H})$, $4.69 \sim 4.75(\mathrm{~m}, 1 \mathrm{H}), 7.12 \sim 7.26(\mathrm{~m}, 7 \mathrm{H}), 7.59(\mathrm{dd}, J=8.9$, $5.0 \mathrm{~Hz}, 2 \mathrm{H}), 7.96(\mathrm{~d}, J=7.0 \mathrm{~Hz}, 1 \mathrm{H}), 8.11(\mathrm{~d}, J=7.9 \mathrm{~Hz}$, $1 \mathrm{H}, \mathrm{NH}), 10.03(\mathrm{~s}, 1 \mathrm{H}, \mathrm{NH}) ;{ }^{13} \mathrm{C} \mathrm{NMR}(100 \mathrm{MHz}$, DMSO- $\left.d_{6}\right) \delta: 173.00,172.57,170.11,159.74,157.36$, $155.99,137.87,135.62,126.86,121.53,115.83,115.61$, $67.45,55.18,50.40,48.81,37.99,22.49,18.52,18.44$, 8.93; IR (KBr) v: 3262, 2980, 1684, 1668, 1641, 1541, $1260,1217,834,700 \mathrm{~cm}^{-1}$. Anal. calcd for $\mathrm{C}_{25} \mathrm{H}_{31} \mathrm{~N}_{4}: \mathrm{C}$ 61.72, H 6.42, N 11.52; found C 61.74, H 6.41, N 11.55.

4-三氟甲氧基苯胺- $L$-苯丙氨酰- $L$-丙氨酰- $L$-丙氨酰 异丙酯(以 $7 \mathrm{~b}$ 和 $1 \mathrm{~b}$ 为原料)(8b): 白色粉末固体, 纯度 $98.5 \%$, 收率 $66.8 \%$. m.p. $223.2 \sim 223.7{ }^{\circ} \mathrm{C}$; $[\alpha]_{\mathrm{D}}^{20}+10.2$ (c 1.0, DMF); ${ }^{1} \mathrm{H}$ NMR (400 MHz, DMSO- $\left.d_{6}\right) \delta: 1.15(\mathrm{t}$, $J=7.5 \mathrm{~Hz}, 12 \mathrm{H}), 2.91 \sim 2.96(\mathrm{~m}, 2 \mathrm{H}), 3.06 \sim 3.11(\mathrm{~m}, 1 \mathrm{H})$, $3.97 \sim 4.03(\mathrm{~m}, 1 \mathrm{H}), 4.22 \sim 4.27(\mathrm{~m}, 1 \mathrm{H}), 4.59 \sim 4.64(\mathrm{~m}$, $1 \mathrm{H}), 4.69 \sim 4.75(\mathrm{~m}, 1 \mathrm{H}), 7.18 \sim 7.32(\mathrm{~m}, 7 \mathrm{H}), 7.70(\mathrm{~d}, J=$ $8.8 \mathrm{~Hz}, 2 \mathrm{H}), 7.97$ (d, $J=6.8 \mathrm{~Hz}, 1 \mathrm{H}, \mathrm{NH}), 8.16$ (d, $J=7.4$ $\mathrm{Hz}, 1 \mathrm{H}, \mathrm{NH}), 10.24$ (s, 1H, NH); ${ }^{13} \mathrm{C} \mathrm{NMR}(100 \mathrm{MHz}$, DMSO- $\left.d_{6}\right) \delta: 173.02,172.65,170.48,155.99,144.13$, 138.42 , 137.81, 129.62, 128.59, 126.89, 122.07, 121.15, $119.34,67.44,55.26,50.37,48.78,37.88,22.49,18.53$, 18.44, 9.51; IR (KBr) v: 3294, 2982, 1683, 1651, 1540, $1267,1224,845,700 \mathrm{~cm}^{-1}$. Anal. calcd for $\mathrm{C}_{26} \mathrm{H}_{31} \mathrm{~N}_{4}$ : C 
56.52, H 5.65, N 10.14; found C 56.54, H 5.64, N 10.16; HRMS calcd for $\mathrm{C}_{26} \mathrm{H}_{31} \mathrm{~F}_{3} \mathrm{~N}_{4} \mathrm{NaO}_{6}[\mathrm{M}+\mathrm{Na}]^{+}$575.2098, $[2 \mathrm{M}+\mathrm{Na}]^{+}$1127.4298; found $[\mathrm{M}+\mathrm{Na}]^{+}$575.2094, $[2 \mathrm{M}+\mathrm{Na}]^{+} 1127.4259$.

\subsection{MTT 法测试化合物抗肿瘤活性}

本文采用噻唑蓝(MTT)比色法测定目标化合物的体 外抗肿瘤活性. 选取对数生长期的人肝癌细胞 SMC7721, 接种到 96 孔板, 每孔约 5000 个细胞, 将化 合物设定 4 个对照浓度分别为 $0.5,1,2,4 \mu \mathrm{mol} / \mathrm{mL}$, 用 酶联免疫检测仪测得各组样品的吸光度值 $(A)$ 后, 按下 列公式对肝癌细胞 SMC7721 增殖的抑制率进行计算:

$$
\text { 抑制率 }(\%)=(A \text { 空白一 } A \text { 加药组 }) / A \text { 空白 } \times 100 \%
$$

致谢 本文中的抗肿瘤活性测试工作委托哈尔滨医科 大学附属肿瘤医院安巍巍老师完成, 特此致谢.

辅助材料(Supporting Information) 所合成目标化合 物的红外、核磁共振氢谱、核磁共振碳谱及高分辨质谱 谱图. 这些材料可以免费从本刊网站(http://sioc- journal.cn/)上下载.

\section{References}

[1] He, P.-J.; Ni, S.-X.; Wang, W.-Q, Jia, J.-B. Chin. Med. Biotechnol. 2009, 4, 288 (in Chinese).

(何平均, 倪淑欣, 王文权, 贾晋斌, 中国医药生物技术, 2009, 4, 288.)

[2] Wang, J.; Xu, Q.-L.; Li, J.-M.; Zhang, E.-L.; Hu, M.-H.; Ye, W.-F.;
Huang, W.-J. Chin. J. Org. Chem. 2014, 34, 2040 (in Chinese). (王杰，许勤龙，李家明，张恩立，胡敏华，叶文峰，黄伟军，有 机化学, 2014, 34, 2040.)

[3] Yin, P.; Hu.-M.-L.; Yan, X.-W.; Wang, Y.; Miu, Q.; Chang, X.-Q. Acta Chim. Sinica. 2008, 57, 1693 (in Chinese).

(尹坪，胡茂林，严小威，王舜，缪谦，常雪琴，化学学报，2008, $57,1693$.

[4] Yan, A,-X.; Tian, G.-L.; Ye, Y.-H. Chin. J. Org. Chem. 2000, 20, 299 (in Chinese)

(间爱新，田桂玲，叶蕴华，有机化学, 2000, 20, 299.)

[5] Petrova, E. E.; Valyakina, T. I.; Komaleva, R. L. Bull. Exp. Biol. Med. 2007, 143, 251.

[6] Reardon, D. A.; Neller, M. Future Oncol. 2011, 7, 339.

[7] Eagle, H. Science 1955, 122, 501.

[8] McCoy, T. A.; Maxwell, M.; Neuman, R. E. Cancer Res. 1956, 16, 979.

[9] Li, X.-Z.; Zhao, P.; Zhan, X.-P.; Liu, Z.-L.; Mao, Z.-M. Chin. J. Org. Chem. 2015, 35, 167 (in Chinese).

(李衍忠, 赵萍, 詹晓萍, 刘增路, 毛振民, 有机化学, 2015, 35, 167.)

[10] Wang, Q.-D.; Xue, S.-J.; Shen, J.-F.; Cai, Z.-J. Chin. J. Org. Chem. 2008, 28, 521 (in Chinese).

(王庆东, 薛思佳, 申杰峰, 蔡志娟, 有机化学, 2008, 28, 521.)

[11] Mishra, S.; Hamburger, A. W. Cancer Res. 1993, 53, 557.

[12] Raida, L.; Rusinakova, Z.; Faber, E.; Szotkowska, R; Rohon, P.; Skoumalova, L.; Divoka, M.; Pikalova, Z.; Indrak, K.; Langova, K. Int. J. Hematol. 2014, 100, 6, 582.

[13] Zhang, J.; Zhang, Y.-M.; Shan, Y.-Y.; Li, N.; Ma, W.; He, L.-C. Eur. J. Med. Chem, 2010, 45, 2798.

[14] Liu, A.-C.; He, X.-L.; Feng, J.-L.; Chen, L.; Zou, X.-D. World Pestic. 2014, 36, 5 (in Chinese).

(刘安昌, 贺晓璐, 冯佳丽, 陈露, 邹晓东, 世界农药, 2014, 36, 5.)

[15] Keller, O.; Keller, W. E.; Look, G. V.; Wersin, G. Org. Synth. 1985, 63, 160 . 